\title{
Corela
}

Cognition, représentation, langage

HS-16 | 2015

Diversité des pratiques de recherche en science du langage

\section{Structuration sémantique du lexique verbal en français langue étrangère chez des apprenants polonais}

Dominika Jagielska

\section{(2) OpenEdition}

\section{Journals}

Édition électronique

URL : http://journals.openedition.org/corela/3819

DOI : $10.4000 /$ corela.3819

ISSN : 1638-573X

\section{Éditeur}

Cercle linguistique du Centre et de l'Ouest - CerLICO

\section{Référence électronique}

Dominika Jagielska, « Structuration sémantique du lexique verbal en français langue étrangère chez des apprenants polonais », Corela [En ligne], HS-16 | 2015, mis en ligne le 29 juin 2015, consulté le 01 mai 2019. URL : http://journals.openedition.org/corela/3819; DOI : 10.4000/corela.3819

Ce document a été généré automatiquement le 1 mai 2019.

\section{c) (1)(2)}

Corela - cognition, représentation, langage est mis à disposition selon les termes de la licence Creative Commons Attribution - Pas d'Utilisation Commerciale - Partage dans les Mêmes Conditions 4.0 International. 


\title{
Structuration sémantique du lexique verbal en français langue étrangère chez des apprenants polonais
}

\author{
Dominika Jagielska
}

\section{Introduction}

$1 \quad$ Notre objectif est double; nous voulons à la fois étudier la structuration hiérarchique des verbes et révéler la présence d'énoncés non conventionnels à pivot verbal au cours de l'acquisition d'une langue étrangère.

2 Lors de l'acquisition d'une langue étrangère, on observe un recours massif à des verbes génériques ; ce phénomène est mis en relief par différents auteurs tels que Viberg (2002), Noyau $(2005 ; 2008)$ et Bernicot $(1981)$. Ces verbes s'appliquent à de nombreux domaines et à de nombreux objets ; par exemple, le verbe couper s'applique à des objets à dimension solide, et cela de différentes manières, car il s'utilise avec des objets en papier, en tissu mais aussi en bois, en brique, en porcelaine, en plastique ou en verre. À l'inverse, un verbe spécifique ne peut s'étendre à des objets de natures différentes qui renvoient à des dimensions sémantiques variées. Par exemple, le verbe scier peut s'appliquer au bois mais pas au papier, au tissu, à la porcelaine ou au verre. Un verbe spécifique peut comporter l'objet ou l'instrument de l'action, comme déshabiller qui comprend l'objet « habit », ou scier qui contient l'instrument « scie ». Les verbes génériques, du type faire, mettre, casser, couper, enlever seraient acquis et utilisés avant les verbes spécifiques, du type scier, écorcer, briser, découdre.

3 Il s'agit de souligner l'existence, dans les productions des participants, de verbes génériques au sein d'énoncés conventionnels (exemple (1) ci-dessous) mais aussi non conventionnels (exemple (2) ci-dessous) :

(1) - « Elle coupe le persil », pour l'action /découper persil/, (enfant de 4 ans)

(2) - « Elle épile l'orange », pour l'action / peler orange /, (adulte) 
Les énoncés non conventionnels sont classifiés en différentes catégories dans la littérature. Winner (1978: 469) en propose trois sortes :

- Premièrement, un énoncé non conventionnel est considéré comme une erreur ;

- Deuxièmement, dès lors qu'il y a décalage entre deux ou plusieurs noms dans la phrase, ou entre plusieurs idées, on emploie le terme de métaphore (Ricœur, 1975) ;

- Enfin, il est question de la notion de sur-extension à propos des énoncés incorrects des enfants. Par exemple, lorsqu'un enfant de deux ans utilise le mot chien pour désigner un chat, un mouton ou un cheval, il fait une sur-extension (Clark, 2003). Il désigne par ce biais un mammifère à quatre pattes. L'hypothèse la plus probable est qu'il ne connaît pas le mot approprié, alors il choisit un terme proche plus accessible. En effet, Gelman (1998), et avec lui Clark (2003), Markman, Merriman \& Bowman, Mervis \& Bertrand (cités in Gelman, 1998), remarquent que les enfants font davantage de sur-extensions quand ils ne connaissent pas le mot de départ.

5 Un autre éclairage de ces énoncés non conventionnels est de les considérer comme des approximations sémantiques fondées sur une relation lexico-sémantique. Le terme d'approximation a été proposé par Jakobson en 1956. Pour cet auteur les approximations seraient des «identifications approchées [...] de nature métaphorique ». Cette position suggère de voir dans ces productions non conventionnelles la marque d'une flexibilité cognitive, à l'œuvre non seulement chez le locuteur durant le développement lexical de sa langue maternelle, mais aussi chez le locuteur en situation d'acquisition d'une langue étrangère (Duvignau, Gaume \& Nespoulous, 2004 ; Duvignau, Gardes-Tamine \& Gaume, 2004). Cette flexibilité, primordiale dans le développement du lexique, permettrait de pallier les manques de mots aussi bien chez l'enfant acquérant la langue que chez l'adulte ou l'enfant apprenant une langue étrangère. Selon Duvignau (2003), un énoncé non conventionnel à pivot verbal refléterait un mode d'organisation des verbes par cohyponymie. Les verbes sélectionnés seraient en réalité co-hyponymes des verbes conventionnels attendus. L'auteur lie la notion d'approximation au couple hyperonymiehyponymie.

Dans notre étude, notre hypothèse est que, loin d'être des énoncés erronés, les énoncés non conventionnels à pivot verbal (incluant ou non des verbes génériques) se révèlent être des productions essentielles dans le développement lexical qui permettent de dépasser une situation de faiblesse lexicale ou de problème d'accès au lexique. À cette fin, nous postulons que nos sujets utilisent un nombre important de verbes génériques, notamment dans un contexte non conventionnel.

7 Dans un premier temps, nous présenterons nos participants ainsi que la méthodologie utilisée pour recueillir et classifier nos résultats en termes de verbes génériques et d'énoncés non conventionnels. Dans un second temps, nous fournirons quelques données statistiques. Enfin, nous exposerons nos analyses qualitatives.

\section{Méthodologie}

\subsection{Participants}

8 Nos participants ont été sélectionnés selon plusieurs critères. Chacun devait avoir pour langue maternelle le polonais et être en cours d'apprentissage du français. Nous avons sélectionné des enfants et des adultes dont le niveau en français était introductif (au 
maximum un an d'apprentissage) ou intermédiaire (plus d'un an d'apprentissage) et nous leur avons fait passer un protocole expérimental. Nous dénombrons au total vingt-quatre participants, interrogés dans plusieurs villes de France (Strasbourg, Paris, Dijon) et de Pologne (Varsovie). Notre population se compose de :

- 7 enfants de 3-5 ans (en acquisition précoce du langage) ;

- 4 enfants de 6-10 ans (acquisition tardive) ;

- 13 adultes.

9 Ces participants sont répartis selon leur âge et niveau en français :

- Enfants en acquisition précoce et de niveau introductif en français: FLE-EAP-NI 1 à 7 (groupe A) ;

- Enfants en acquisition tardive et de niveau introductif en français : FLE-EAT-NI 1 à 4 (groupe B) ;

- Adultes de niveau introductif en français : FLE-AD-NI 1 à 6 (groupe C) ;

- Adultes de niveau intermédiaire en français : FLE-AD-NIM 1 à 7 (groupe D) ;

Nous avons choisi de comparer deux populations d'enfants: enfants en acquisition précoce et enfants en acquisition tardive. Dans nos résultats, nous présentons les participants par leur codage (FLE-EAP-NI 2 par exemple).

\subsection{Protocole expérimental : «Approx»}

11 Pour révéler les énoncés non conventionnels et verbes génériques, les participants de cette étude ont été soumis à un protocole, il s'agit d'« Approx » (Duvignau \& Gaume, 2001, 2004). Ce dernier a été réalisé dans le but d'être présenté à différents types de participants : de langues maternelles diverses; participants présentant des pathologies dont l'autisme ou le syndrome d'Asperger. Le protocole consiste à regarder une vidéo montrant une succession de dix-sept actions d'ordre quotidien exécutées par une femme.

On considère trois catégories d'action : « enlever », « séparer » et « détériorer » :

\begin{tabular}{|c|c|c|}
\hline DETERIORER & ENLEVER & SEPARER \\
\hline $\begin{array}{l}\text { 1. /briser verre/ } \\
\text { (avec un marteau) } \\
\text { 2. /déchirer journal/ } \\
\text { 8. /éclater ballon/ } \\
\text { 10. /écraser tomate/ } \\
\text { (avec la main) } \\
\text { 13. /froisser papier/ }\end{array}$ & $\begin{array}{l}\text { 6. /démonter legos/ } \\
\text { 7. /déshabiller poupée/ } \\
\text { 9. /écorcer arbre/ } \\
\text { (l'écorce d'une bûche) } \\
\text { 12. /éplucher carotte/ } \\
\text { (avec un économe) } \\
\text { 14. /peler banane/ } \\
\text { (avec les mains) } \\
\text { 15. /peler orange/ } \\
\text { (avec les mains) }\end{array}$ & $\begin{array}{l}\text { 3. /découdre chemise/ } \\
\text { 4. /découper pain/ } \\
\text { (avec un couteau) } \\
\text { 5. /hacher persil/ } \\
\text { (avec un couteau) } \\
\text { 11. /émietter pain/ } \\
\text { (avec les mains) } \\
\text { 16. / rompre pain/ } \\
\text { (avec les mains) } \\
\text { 17. /scier planche/ }\end{array}$ \\
\hline
\end{tabular}

Tableau 1 - Présentation des actions du protocole expérimental

L'ensemble des actions est destiné à être présenté en mode aléatoire avec néanmoins la consigne de passer systématiquement le film /éclater ballon/ en dernier. Cette contrainte 
est prévue pour la population Asperger et autiste car la brusquerie de cette action peut entraîner de vives réactions de la part de cette population. Il est demandé de la passer en dernier afin de ne pas compromettre le déroulement de la passation. Nous respectons cette contrainte pour nos participants.

Dans cette étude, nous exposons des résultats pour chaque vidéo du protocole. Suite au visionnage de chacun de ces films, nous avons proposé deux tâches aux participants. La première est une tâche de dénomination d'action avec la consigne suivante, donnée durant la réalisation de l'action cible : "Qu'est ce qu'elle a fait la dame? ?.

Cette première tâche est suivie d'une tâche de reformulation d'action. Le film n'est plus visible, nous demandons alors : "Ce qu'elle a fait la dame, dis-le moi d'une autre manière, avec d'autres mots». Nous notons la réponse entière à chaque fois. Nous présentons ici des résultats issus de ces tâches de dénomination et de reformulation.

\subsection{Codage des réponses}

16 Nous classons nos résultats selon les critères valide/invalide, conventionnel/ approximatif et générique/spécifique.

Une réponse est considérée comme valide lorsqu'elle comporte un verbe pouvant se relier avec pertinence à l'action-cible à dénommer, comme peler orange pour /éplucher orange/ par exemple. À l'inverse, est considérée comme invalide une réponse qui ne comporte pas de verbe pouvant se relier avec pertinence à l'action-cible à dénommer, comme Elle va manger une orange pour /éplucher orange/.

18 Un verbe est dit conventionnel lorsqu'il appartient au même champ d'application lexicoconceptuel que celui du nom avec lequel il se combine dans l'énoncé. Son usage ne comporte aucune tension, c'est-à-dire aucune équivoque, ni sémantique ni pragmatique, et peut être dès lors considéré comme habituel, conventionnel, dans le système linguistique concerné, tels les verbes éplucher, peler, enlever pour /éplucher orange/.

Un verbe est dit approximatif lorsque son usage provoque une tension. Deux types de tension sont identifiés :

- Le premier type de tension concerne le lien entre le verbe et le nom. L'approximation est considérée extra-domaine quand deux domaines sémantiques sont en jeu. Par exemple, l'emploi de verbes comme déshabiller, plumer, casser ou épiler pour l'action /éplucher orange/ crée un conflit verbe-objet. Néanmoins, les verbes impliqués sont en relation de synonymie intra-domaine, ou co-hyponymie extra-domaine, car ils partagent un même hyperonyme. Ainsi, peler et déshabiller sont des synonymes ou co-hyponymes extra-domaines qui partagent le même hyperonyme « enlever ».

- Le second type de tension est observé entre le verbe et la réalité désignée. Le verbe appartient au même domaine sémantique que le nom en jeu dans le film, mais ne désigne pas l'action en jeu, comme couper pour /éplucher orange/ par exemple. Dans ce cas, l'approximation est intra-domaine puisque seul un domaine sémantique est impliqué et que la tension est pragmatique. Les verbes choisis, génériques ou non, partagent une proximité moyenne avec le verbe attendu.

Est générique un verbe qui s'applique à de nombreux domaines et objets, comme couper qui s'applique au papier, tissu, verre, etc. À l'inverse, un verbe est spécifique lorsqu'il comporte dans sa morphologie l'objet auquel il renvoie, ou bien l'instrument, ou encore 
le résultat impliqué dans l'action concernée, c'est le cas de scier qui comporte l'instrument scie.

Dans notre étude, nous ne prenons en considération que les réponses valides et parmi celles-ci celles qui comportent des verbes génériques, soit en emploi conventionnel, avec une proximité forte entre le verbe énoncé et le verbe cible, soit en emploi approximatif, avec une proximité moyenne entre le verbe énoncé et le verbe cible.

Dans la partie suivante nous présentons les analyses statistiques effectuées à partir de nos résultats.

\section{Résultats statistiques}

Nous avons retenu les réponses valides/invalides, les verbes génériques/spécifiques et les énoncés approximatifs/conventionnels. Pour chaque étude de groupe ou de comparaison de groupes, nous prenons en considération ces éléments. Pour chacun d'eux, les calculs ont été faits par rapport à l'ensemble des réponses sur les 17 films.

Nous considérons ainsi :

- Les réponses valides par rapport aux réponses invalides ;

Les deux éléments suivants concernent les réponses valides :

- Les approximations par rapport aux énoncés conventionnels ;

- Les verbes génériques par rapport aux verbes spécifiques (comme le calcul est effectué sur l'ensemble des réponses, soit des énoncés conventionnels soit des approximations, les verbes génériques et spécifiques sont considérés dans un emploi conventionnel et approximatif).

Nous donnons le pourcentage de réponses pour chacun de ces éléments et nous comparons ces pourcentages selon les paires considérées (valide/invalide; générique/ spécifique ; approximation/conventionnel) :

\begin{tabular}{|l|l|l|l|l|l|l|}
\hline Groupe & $\begin{array}{l}\% \\
\text { réponses } \\
\text { valides }\end{array}$ & $\begin{array}{l}\% \\
\text { réponses } \\
\text { invalides }\end{array}$ & $\begin{array}{l}\% \\
\text { verbes } \\
\text { génériques }\end{array}$ & $\begin{array}{l}\% \\
\text { verbes } \\
\text { spécifiques }\end{array}$ & $\begin{array}{l}\% \\
\text { approximations }\end{array}$ & $\begin{array}{l}\% \\
\text { énoncés } \\
\text { conventionnels }\end{array}$ \\
\hline $\begin{array}{l}\text { Groupe } \\
\text { A }\end{array}$ & $49 \%$ & $51 \%$ & $37 \%$ & $12 \%$ & $24 \%$ & $25 \%$ \\
\hline $\begin{array}{l}\text { Groupe } \\
\text { C }\end{array}$ & $30 \%$ & $70 \%$ & $14 \%$ & $16 \%$ & $9 \%$ & $21 \%$ \\
\hline $\begin{array}{l}\text { Groupe } \\
\text { D }\end{array}$ & $97 \%$ & $3 \%$ & $21 \%$ & $76 \%$ & $16 \%$ & $81 \%$ \\
\hline
\end{tabular}

Tableau 2 - taux et pourcentages pour les groupes en français langue étrangère

Le groupe B ne figure pas dans les analyses statistiques pour des raisons d'homogénéité.

Nous constatons que le groupe $\mathrm{C}$ produit le moins de réponses valides au test.

Nous observons une forte production de verbes génériques par tous nos sujets en acquisition d'une langue étrangère. Le groupe A est le groupe qui en produit le plus, aussi 
bien en termes de pourcentage qu'en termes de proportion (deux tiers des réponses valides). En termes de proportion, il est suivi du groupe C (la moitié des réponses valides) puis du groupe $\mathrm{D}$ (un cinquième des réponses valides). En acquisition d'une langue étrangère, la population « enfants en acquisition précoce du langage » est susceptible de produire un pourcentage significatif de verbes génériques, ce qui est confirmé par les analyses statistiques. De même, les adultes de niveau introductif en produisent un taux significatif. Les adultes de niveau intermédiaire conservent un taux notable de verbes génériques.

Concernant la production d'approximations, nous observons qu'elle est plus élevée pour le groupe A. Cela témoigne d'une production d'approximations supérieure chez les enfants par rapport aux adultes FLE. En termes de proportion, le groupe A produit le plus d'approximations (environ la moitié des réponses valides), suivi du groupe $\mathrm{C}$ (un tiers des réponses valides) et enfin du groupe $\mathrm{D}$ (moins d'un cinquième des réponses valides).

Nous constatons que le groupe A produit le plus fort pourcentage de verbes génériques et d'approximations des trois groupes FLE comparés. Nous pouvons en conclure que les enfants en acquisition précoce du langage produisent une quantité supérieure de verbes génériques et d'approximations par rapport à d'autres profils de sujets en acquisition d'une langue étrangère.

\section{Résultats : analyses qualitatives}

Dans cette partie, nous présentons nos résultats selon deux critères : verbe générique et approximation. Nous donnons ainsi l'exemple d'un verbe générique, casser, qui s'applique à divers domaines et objets, énoncé dans un emploi approximatif. Dans ce type d'énoncés, il existe un conflit sémantique entre le verbe et l'objet, ou un décalage entre le verbe et la réalité désignée. Toutefois, l'énoncé ne peut être qualifié d'erroné puisque le verbe choisi partage avec le verbe attendu une proximité sémantique moyenne. Observons notre exemple 3 :

(3) : le verbe casser

Nous relevons une utilisation importante du verbe casser. En effet, la plupart des participants utilisent ce verbe en emploi approximatif pour plusieurs actions. L'emploi n'est pas considéré comme conventionnel parce qu'il existe un décalage entre le verbe et les objets de l'action ou encore avec la situation de référence. Néanmoins, les réponses sont de nature valide puisque le verbe cité possède des traits communs à ceux du verbe attendu. Nous avons mentionné auparavant que lorsque deux domaines sémantiques sont en jeu, il s'agit d'approximations extra-domaines et lorsqu'il est question d'un décalage entre le verbe et la situation de référence, il s'agit d'approximations intra-domaines. 
Pour l'action /déchirer journal/ :

f. FLE-EAT-NI 3 : Elle casse

g. FLE-EAP-NI 1 : Elle a cassé une gazette

Pour l'action /rompre pain/ :

h. FLE-AD-NIM 3 : Casser le pain

i. FLE-EAT-NI 3 : Elle a cassé en deux

Pour l'action /découdre chemise/ :

j. FLE-EAP-NI 1 : C'est une chemise. Popsuła. Elle a cassé

k. FLE-EAP-NI 4 : Elle casse des boutons

Soit les exemples suivants en tâches de reformulation :

Pour l'action /éclater ballon/ :

1. FLE-EAT-NI 3 : Elle a cassé le ballon

Pour l'action /froisser papier/:

m. FLE-EAT-NI 3 : Elle a cassé, non pas casser

n. FLE-EAP-NI 2 : Elle a cassé quelque chose. Elle a fait une boule

Pour l'action /scier planche/:

o. FLE-EAT-NI 3 : Elle a cassé la planche

Pour l'action /émietter pain/ :

p. FLE-EAP-NI 4 : Elle a cassé le manger

Pour l'action /peler orange/ :

q. FLE-EAT-NI 3 : Elle enlève la peau, elle a cassé

Nous observons qu'une tension se profile entre le verbe et les objets de l'action : un heurt sémantique apparaît entre casser et, par exemple, ballon ou tomate. Le verbe casser a plusieurs significations selon le Petit Robert (2011) : « Mettre en morceaux, diviser (une chose rigide) d'une manière soudaine, par choc, coup, pression » ou encore « Endommager, empêcher son fonctionnement ». La combinaison du verbe casser avec des objets comme ballon, tomate ou papier paraît erronée à première vue car ces objets (du type tissu, aliment, ou papier) ne sont pas de consistance solide et ne possèdent pas non plus de système de fonctionnement. Toutefois, le verbe exprime l'idée de destruction et est utilisé en majorité par les participants dans les actions correspondant à la détérioration. Il est également employé dans les catégories d'action "séparer » et « enlever». En définitive, le verbe est énoncé pour des actions correspondant aux trois catégories, « détériorer ", " séparer » et « enlever », ce qui mérite d'être souligné.

Casser est co-hyponyme extra-domaine de froisser, éclater, déchirer et écraser : ils partagent l'hyperonyme « détériorer ».

Casser est co-hyponyme extra-domaine de découdre, rompre, émietter et scier : ils partagent l'hyperonyme « séparer».

Casser est co-hyponyme extra-domaine de peler : ils partagent l'hyperonyme « enlever ».

Casser est un verbe très utilisé par tous les participants, de plus il est employé pour toutes les catégories d'action. Les verbes comme casser sont accessibles et fréquents parce qu'ils font référence à des situations familières et qu'ils s'appliquent à de nombreuses situations de la vie quotidienne. Les participants, même s'ils présentent un manque de lexique, ont par conséquent accès à ce type de verbe, et ils l'utilisent pour des contextes variés. Ils n'appliquent pas toujours ces verbes de manière conventionnelle mais néanmoins leur 
utilisation n'est pas erronée. En définitive, toutes les catégories de participants, adultes comme enfants, produisent des approximations sémantiques basées sur le sémantisme de casser. Ce type de verbe est accessible à tout type de population en acquisition d'une langue étrangère.

Dans le « Français Fondamental » (Gougenheim et al., 1964), casser figure à la $770^{\text {ème }}$ place du classement de la fréquence d'emploi des mots et fait partie des 1063 mots les plus fréquemment utilisés. Selon cette étude, le verbe casser est très fréquent dans la langue parlée alors que ses synonymes, briser et rompre, sont plus courants dans la langue écrite.

\section{Bilan et Perspectives}

Notre étude se proposait de mettre en relief l'emploi de verbes génériques, en français langue étrangère, pour une population d'enfants et d'adultes. Un type d'usage a été mis en valeur : un usage non conventionnel. Nos participants utilisent des verbes génériques en emploi non conventionnel. Le verbe énoncé partage alors une proximité moyenne avec le verbe cible.

Bernicot (1981), Viberg (2002) et Noyau $(2005 ; 2008)$ soulignent la prédominance de verbes génériques du type faire, dire, prendre ou voir, au cours de l'acquisition d'une langue maternelle comme d'une langue étrangère. Ils appartiennent à des domaines sémantiques dont ils sont en quelque sorte les représentants. Ainsi, le verbe faire pourrait être considéré comme le verbe phare des verbes d'activité. Slobin (cité in Bruner, 1987) suggère qu'il y a des modes types par lesquels l'enfant fait l'expérience du monde, par exemple un évènement transitif type. De ce fait, un verbe comme casser, largement utilisé par nos participants, pourrait correspondre à un évènement de détérioration type que les participants en langue étrangère emploient avant d'enrichir leur lexique. Le verbe pourrait représenter la typicité de la détérioration. Les verbes génériques sont des verbes fréquents, des verbes de base rapidement acquis par rapport à des verbes moins fréquents et confinés à un certain type d'actions.

L'utilisation des verbes génériques dans différents contextes par une population fait état de la flexibilité sémantique du lexique des verbes. Les participants sont capables de substituer un verbe à un autre de manière à produire des énoncés intelligibles. Par exemple, casser est étendu à diverses actions, il est ouvert à divers contextes, et les sujets se permettent de l'étendre encore davantage. Enfants et adultes tiennent compte ou négligent certains sèmes (Le Ny, 1987), ce qui est le cas dans le choix de verbes qui contiennent le sème de "détérioration ". Le verbe utilisé possède un vaste champ d'application ce qui rend possible son utilisation pour des actions auxquelles il n'est pas à priori approprié. Les concepts que maitrisent les participants déterminent leur choix de mots : ils choisissent un verbe qui répond au concept, qui y est inclus, comme casser est inclus dans le concept de détérioration.

Gentner (1978) rejoint ces théories, et affirme que, par l'utilisation des verbes, les enfants démontrent une capacité à faire des analogies et des extensions créatives. Or cette capacité s'observe également chez nos apprenants du français langue étrangère: ils étendent le verbe à différentes situations et décident de choisir un verbe quand seulement une partie de son sens s'applique. Ils baseraient ainsi le sens d'un nouveau mot sur les données conceptuelles qu'ils possèdent; ces données conceptuelles seraient liés à 
leur culture et à leur apprentissage au sein d'une société particulière, chaque langue possédant ses concepts propres.

La valeur de vérité d'une proposition est inaltérée quand on substitue à une expression donnée une expression de même dénotation (Frege, 1971). Par le choix d'un verbe non conventionnel, les participants rendent compte de la même réalité qu'avec l'utilisation d'un verbe approprié.

En outre, les verbes choisis se révèlent être co-hyponymes des verbes attendus. Ainsi casser, utilisé pour l'action /froisser papier/, est co-hyponyme extra-domaine de froisser: ils partagent l'hyperonyme "détériorer ». Nous proposons une organisation du lexique par proximité sémantique, laquelle peut être utilisée pour l'apprentissage d'une langue étrangère.

Utiliser un verbe générique de manière conventionnelle ou non fait appel à des processus qui comptent dans l'acquisition et la structuration du lexique verbal en langue étrangère. Les approximations sémantiques marquent une stratégie palliative au manque de mots.

Selon Bernicot (1981), Viberg (2002) et Noyau $(2005 ; 2008)$, le phénomène de recours à des verbes génériques est à l'œuvre lors de l'acquisition d'une langue maternelle. De même la production d'énoncés non conventionnels, ou approximations sémantiques à pivot verbal, reflet d'une flexibilité cognitive et sémantique, serait importante lors de l'acquisition d'une langue maternelle, comme le postulent Duvignau, Gaume \& Nespoulous (2004). Nous pourrions de ce fait postuler que nos participants produiraient un nombre important de verbes génériques et d'énoncés non conventionnels à pivot verbal en polonais langue maternelle. Ceci pourrait faire l'objet d'un autre article.

\section{BIBLIOGRAPHIE}

Bernicot, Josie (1981), Le développement des systèmes sémantiques de verbes d'action, Paris : Editions du Centre National de la Recherche Scientifique.

Bruner, Jérome. (1987), Comment les enfants apprennent à parler, Col. Actualités pédagogiques, Paris : Retz.

Clark, Eve V. (2003), First Language acquisition, Cambridge : Cambridge University Press.

Duvignau, Karine (2003), « Métaphore verbale et approximation », Revue d'Intelligence Artificielle, Vol 5/6, 869-881.

Duvignau Karine, Gaume, Bruno \& Nespoulous, Jean-Luc (2004), « Proximité sémantique et stratégies palliatives chez le jeune enfant et l'aphasique. » Parole, $\mathrm{n}^{\circ} 31-32,219-255$.

Duvignau, Karine, Gardes-Tamine, Joëlle \& Gaume, Bruno (2004), « Approximations sémantiques enfantines et distance inter-verbes : pour une organisation proxémique du lexique verbal », Le langage et l'homme, $\mathrm{n}^{\circ}$ 39-2, 123-141.

Gelman, Susan A. (1998), "Why is a pomegranate an apple? The role of shape, taxonomic relatedness, and prior lexical knowledge in children's overextensions of apple and dog", Journal of Child Language, $\mathrm{n}^{\circ} 25,267-291$. 
Gentner, Deirdre (1978), "On relational meaning : the acquisition of verb meaning", Child development, $n^{\circ} 49,988-998$.

Gougenheim, Georges, Rivenc, Paul, Michea René \& Sauvageot, Aurélien (1964), L'élaboration du français fondamental ( $1^{\text {er }}$ degré), Paris : Didier.

Le Ny, Jean-François (1979), La sémantique psychologique, Paris : PUF.

Noyau, Colette (2005), « Le lexique verbal dans l'acquisition d'une langue seconde : verbes de base, flexibilité sémantique, granularité », in Grossmann F., Paveau M.-A. \& Petit G. (eds.), Apprentissage du lexique : langue, cognition, discours, Grenoble : ELLUG, 65-84.

Noyau, Colette (2008), « Place des verbes dans le Français Fondamental, acquisition du lexique verbal en français langue seconde, et didactique du lexique. ", in Bouchard R. \& Cortier C., Pratiques et représentations de l'oral en FLES, 50 ans après le français fondamental. Le français dans le Monde-Recherches et applications, $\mathrm{n}^{\circ}$ spécial, 87-101.

Viberg, Ake (2002), "Basic verbs in Second Language Acquisition." Revue Française de Linguistique Appliquée, $\mathrm{n}^{\circ}$ VII-2, 61-79.

Winner, Ellen (1978), "New names for old things : the emergence of metaphoric language", Journal of child Language, $\mathrm{n}^{\circ}$ 6, 469-491.

Le Petit Robert (2011), ISBN : 978-2-84902-767-7.

\section{RÉSUMÉS}

Notre étude porte sur l'acquisition des verbes en contexte d'acquisition du français langue étrangère. En effet, on constate un recours important à des verbes génériques du type casser, couper, faire en acquisition du lexique verbal d'une langue étrangère. Ces verbes peuvent faire l'objet d'emplois non conventionnels. Les énoncés non conventionnels à pivot verbal du type Elle casse une tomate sont classifiés comme des erreurs, des sur-extensions ou encore des métaphores. Cependant, dans la lignée de certains auteurs, nous postulons que ce type d'énoncé est le reflet d'une flexibilité sémantique primordiale au cours de l'acquisition du lexique d'une langue étrangère, notamment du lexique verbal, qui serait structuré par proximité sémantique. Notre population se compose d'enfants et d'adultes polonais apprenant le français.

Our study deals with verbal acquisition in the acquisition of French as a foreign language. We observe that, when acquiring the verbal lexicon of a foreign language, learners use a large number of generic verbs such as to cut, to do, to break. These verbs may be used in non conventional statements. Non conventional statements based on verbs, such as : "she is breaking a tomato" are considered as errors, over-extensions or metaphors. But we assert that these statements reflect a semantic flexibility which is essential to the acquisition of the lexicon when learning a first and second language. This also applies to the verbal lexicon, which we believe is organized through semantic proximity. Our subjects are Polish adults and children. The children may be in the early or late stages of language acquisition. All are learning French.

\section{INDEX}

Mots-clés : verbes, acquisition, français langue étrangère, sémantique, approximation, générique

Keywords : verbs, semantic, generic, French as a foreign language 


\section{AUTEUR}

\section{DOMINIKA JAGIELSKA}

CLLE-ERSS

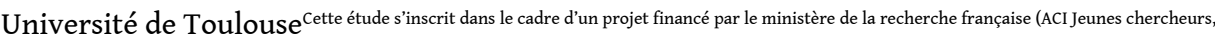

Duvignau (2004)).jagielskadominika@hotmail.com 